\title{
Co-option of EDM2 to distinct regulatory modules in Arabidopsis thaliana development
}

\author{
Tokuji Tsuchiya and Thomas Eulgem*
}

\begin{abstract}
Background: Strong immunity of plants to pathogenic microorganisms is often mediated by highly specific mechanisms of non-self recognition that are dependent on disease resistance $(R)$ genes. The Arabidopsis thaliana protein EDM2 is required for immunity mediated by the $R$ gene RPP7. EDM2 is nuclear localized and contains typical features of transcriptional and epigenetic regulators. In addition, to its role in immunity, EDM2 plays also a role in promoting floral transition. This developmental function of EDM2, but not its role in RPP7-mediated disease resistance, seems to involve the protein kinase WNK8, which physically interacts with EDM2 in nuclei.

Results: Here we report that EDM2 affects additional developmental processes which include the formation of leaf pavement cells and leaf expansion as well as the development of morphological features related to vegetative phase change. EDM2 has a promoting effect of each of these processes. While WNK8 seems not to exhibit any vegetative phase change-related function, it has a promoting effect on the development of leaf pavement cells and leaf expansion. Microarray data further support regulatory interactions between WNK8 and EDM2. The fact that the effects of EDM2 and WNK8 on leaf pavement cell formation and leaf expansion are co-directional, while WNK8 counteracts the promoting effect of EDM2 on floral transition, is surprising and suggests that WNK8 can modulate the activity of EDM2.

Conclusion: We propose that EDM2 has been co-opted to distinct regulatory modules controlling a set of different processes in plant immunity and development. WNK8 appears to modulate some functions of EDM2.
\end{abstract}

\section{Background}

The defense regulator EDM (Enhanced Downy Mildew) 2 was previously shown in Col (Columbia) accessions of Arabidopsis thaliana (Arabidopsis) to be specifically required for immunity mediated by the disease resistance (R)-gene RPP (Resistance to Peronospora parasitica) 7 against the Hiks1 isolate of the pathogenic oomycete Hyaloperonospora arabidopsidis (formerly Peronospora parasitica; Hpa) [1]. Unlike many other plant defense mechanisms RPP7-mediated immunity is independent from the defense hormone salicylic acid. Furthermore, RPP7-mediated immunity appears thus far to be the only defense mechanism that EDM2 is involved in, as no other EDM2-dependent $R$-gene functions have been reported yet. EDM2 also does not contribute to basal defense, a weaker non-specific plant immune response [2]. Thus,

\footnotetext{
* Correspondence: thomas.eulgem@ucr.edu
}

${ }^{1}$ Center for Plant Cell Biology, Institute for Integrative Genome Biology, Department of Botany and Plant Sciences, University of California at Riverside, Riverside, CA 92521, USA

Full list of author information is available at the end of the article the role of EDM2 in plant defense seems to be restricted to a single or a limited number of defense pathways. The EDM2 protein is nuclear-localized and bears typical features of transcription factors and epigenetic regulators, but does not belong to any established class of plant proteins controlling transcription [1,2]. Consistent with a possible role in transcriptional regulation, EDM2 seems to contribute to disease resistance by promoting transcription of RPP7 [1].

We recently reported that a second function of EDM2 is the regulation of flowering time, as edm2 mutants exhibit a delay in floral transition [2]. Consistent with this phenotype, transcript levels of the negative flowering regulator gene FLC (Flowering Locus C) were substantially elevated in $e d m 2$ mutants. As theses edm2 phenotypes are independent from the photoperiod, EDM2 may formally be considered as a member of the autonomous floral promotion pathway, which controls the floral transition by suppressing $F L C$ expression independent from day-length [3].

(c) 2010 Tsuchiya and Eulgem; licensee BioMed Central Ltd. This is an Open Access article distributed under the terms of the Creative BFoMed Central Commons Attribution License (http://creativecommons.org/licenses/by/2.0), which permits unrestricted use, distribution, and reproduction in any medium, provided the original work is properly cited. 
EDM2 interacts in nuclei with the WNK (With No Lysine) 8 protein kinase that can phosphorylate EDM2 [2]. Mutants of this kinase or WNK8 overexpressor lines, however, do not exhibit reduced immunity to HpaHiks1, but $w n k 8$ mutants flower early $[2,4]$. Double mutant analyses showed that EDM2 is epistatic to WNK8. Similarly, $F L C$ proved to be epistatic to EDM2. Thus, WNK8 may act upstream of EDM2 in a regulatory module affecting the floral transition by modulating FLC transcript levels [2]. In this model, WNK8 counteracts the promoting effect of EDM2 on flowering.

Here we report that besides its roles in immunity and the floral transition, EDM2 has several additional functions in Arabidopsis development, such as promoting vegetative leaf growth and leaf pavement cell morphology. WNK8 also has a promoting effect on these processes, suggesting that it participates in these EDM2 functions. However, in contrast to its role in the floral transition, WNK8 seems not to counteract the function of EDM2, but to positively contribute to EDM2 activity. Overlapping transcript profiles of edm2 and $w n k 8$ mutants support common biological roles of both regulators. In addition, EDM2 seems to have a WNK8-independent role in a developmental process related to vegetative phase change. We propose that EDM2 has multiple distinct regulatory functions related to developmental processes and defense. These EDM2 functions differ with respect to their dependency on WNK8. A possible function of WNK8 may be to fine-tune EDM2 activity in some biological processes.

\section{Results}

edm 2 and wnk8 mutants exhibit a reduction in fresh weight The transgenic Arabidopsis line $p X V E: H A-E D M 2-a$ served as a key tool for our previous genetic analysis of EDM2 [2]. This line contains in the edm2-2 mutant background the estradiol-inducible expression construct $p X V E: H A-E D M 2$ encoding the HA epitope tag fused to the $\mathrm{N}$-terminus of the full length EDM2 protein. It exhibits near wild type EDM2 and RPP7 transcript levels in the absence of estradiol [2]. Furthermore, non-estradioltreated $p X V E: H A-E D M 2-a$ plants flower like Col-0 wild type plants and are fully resistant to HpaHiks1, indicating that un-induced expression of the $p X V E: H A-E D M 2$ construct restores wild type $E D M 2$ function. Therefore, we used non-estradiol-treated $p X V E: H A-E D M 2-a$ plants for all complementation experiments described in this study.

Growing edm 2 mutants beyond the seedling stage, we observed multiple distinct morphological phenotypes. By the time of bolting, edm2 plants appeared smaller due to reduced expansion of the rosette leaves and, consequently, exhibited a significant reduction in fresh weight (Figures 1A \&1B). In $p X V E: H A-E D M 2-a$ plants, wild type growth is restored. Mutants of the EDM2-interacting kinase WNK8 also showed a reduction of leaf expansion and fresh weight. However, this effect was only significant in the $w n k 8-1$ and $w n k 8-3$ mutants and less pronounced in the wnk8-2 mutant, which, compared to $w n k 8-1$ and $w n k 8-3$, seems to be a weak allele exhibiting a less pronounced reduction of WNK8 expression [2].

\section{edm2 and wnk8 mutants exhibit altered morphology of leaf pavement cells}

We found the morphology of leaf pavement cells to be altered in $e d m 2$ plants. Typically, the Arabidopsis epidermis consists of interlocked "jigsaw-shaped" cells with protruding lobes and indentations [5,6]. Epidermal cells of edm 2 mutants are typically of simpler morphology often lacking lobes and indentations (Figure 2A). To quantify this effect we measured the average circularity (c), of all cells within a defined leaf area. Circularity is defined as c $=4 \mathrm{pi} \times\left(\right.$ area $/$ perimeter $\left.^{2}\right)$. A perfect circle has a $\mathrm{c}$ value of 1.0, while more complex or elongated shapes have lower $\mathrm{C}$ values due to decreased area:perimeter ratios (Figure 2B). The pronounced jigsaw shapes of Col- 0 epidermis cells are reflected by particularly low c values between 0.1 and 0.2 , while the more oval cells often found in edm2 epidermis have higher c values. As expected, we found the average $\mathrm{c}$ values of each of the four edm 2 mutants to be significantly higher than those of their respective wild type controls (Col-5 for edm2-1; Col-0 for all other lines; Figure $2 C$ ). In $p X V E: H A-E D M 2-a$ plants wild type pavement cell morphology is restored.

Mutations in WNK8 weakly affect the morphology of leaf pavement cells. Like those of $e d m 2$ plants, leaf epidermal cells of $w n k 8$ plants tend to be of simpler morphology often lacking lobes and indentations (Figure 2A). Although all three $w n k 8$ mutants exhibit elevated circularity values of leaf epidermal cells compared to Col-0, the extent of this effect is weaker than in edm 2 mutants and only significant in $w n k 8-1$ (Figure 2C). Stable transformation of the $w n k 8-1$ mutant with a wild type WNK8 transgene containing its native promoter $(w 8-1 / p W 8$ W8) resulted in low circularity values of leaf pavement cells comparable to those in Col-0.

\section{Mutation of EDM2 causes developmental effects related to vegetative phase change}

In Col-0 plants, the first two leaves are small and nearly perfectly round with smooth margins. However, edm2 plants typically form at this point in their development a pair of small leaves that are "spade-shaped" with slight serrations in the leaf margin (Figure 3A). Their shapes resemble the $2^{\text {nd }}$ pair of leaves found in Col-0, but only the shape and not the size is affected. This morphological phenotype suggested altered vegetative phase change in edm2 plants. This developmental process, which is also known as heteroblasty, is defined by changes of leaf anat- 


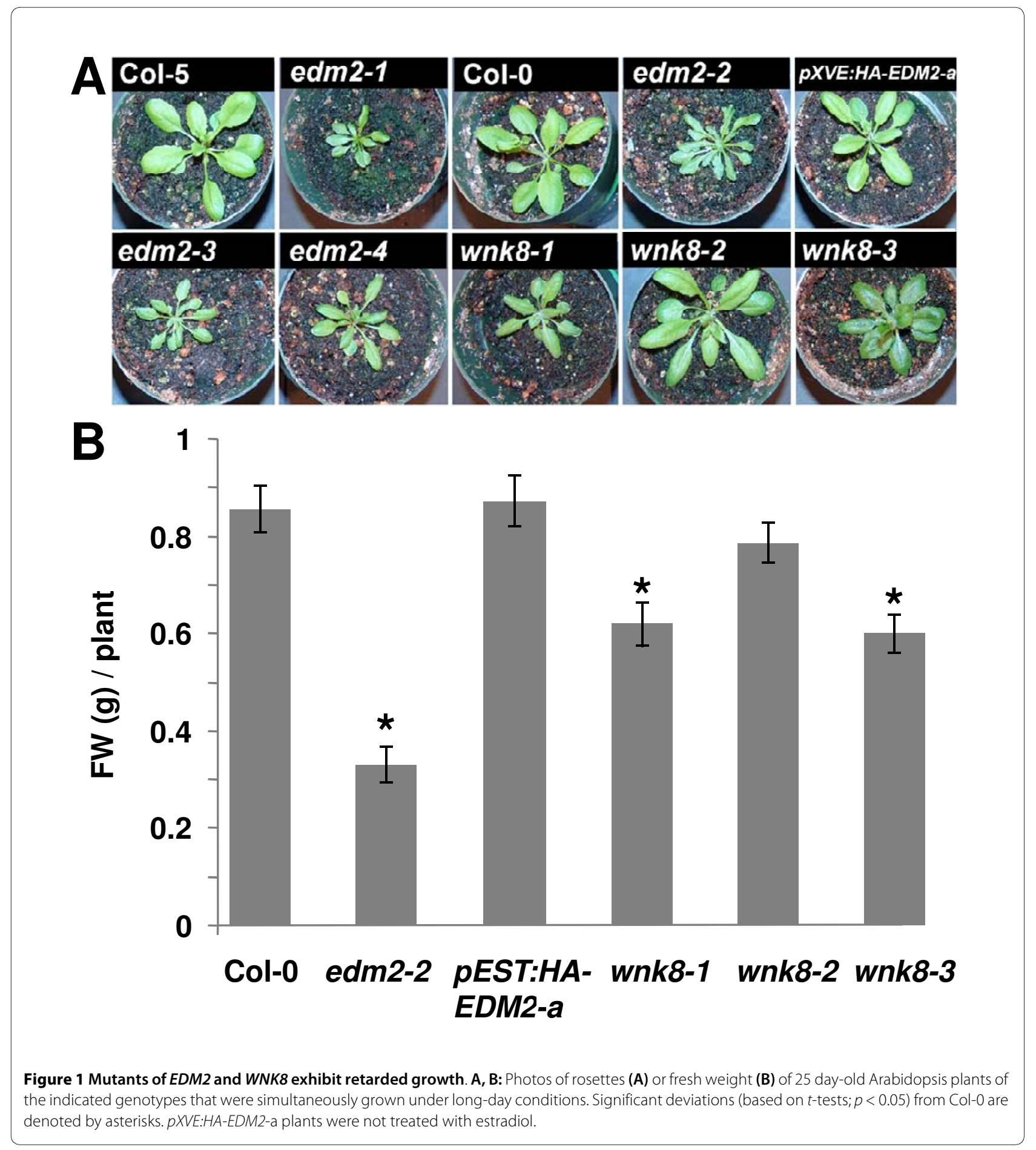

omy and morphology during the vegetative phase of plant ontogeny [7]. Reminiscent of the known Arabidopsis vegetative phase change mutants serrate (se), squint (sqn), hasty (hst), zippy (zip), suppressor of gene silencing 3 (sgs3) and RNA-dependent RNA Polymerase 6 (rdr6) [8-12] the early juvenile phase of vegetative development seemed to be skipped in edm2 plants, which is manifested in an apparent omission of the first pair of regular rosette leaves (Figure 3A).

In order to more accurately measure this effect in $e d m 2-2$, we focused on the production of trichomes as a quantifiable marker of vegetative phase change [13]. Arabidopsis leaves produced during the juvenile phase lack trichomes on their abaxial surface, while the adult phase starts gradually with the production of abaxial trichomes. 

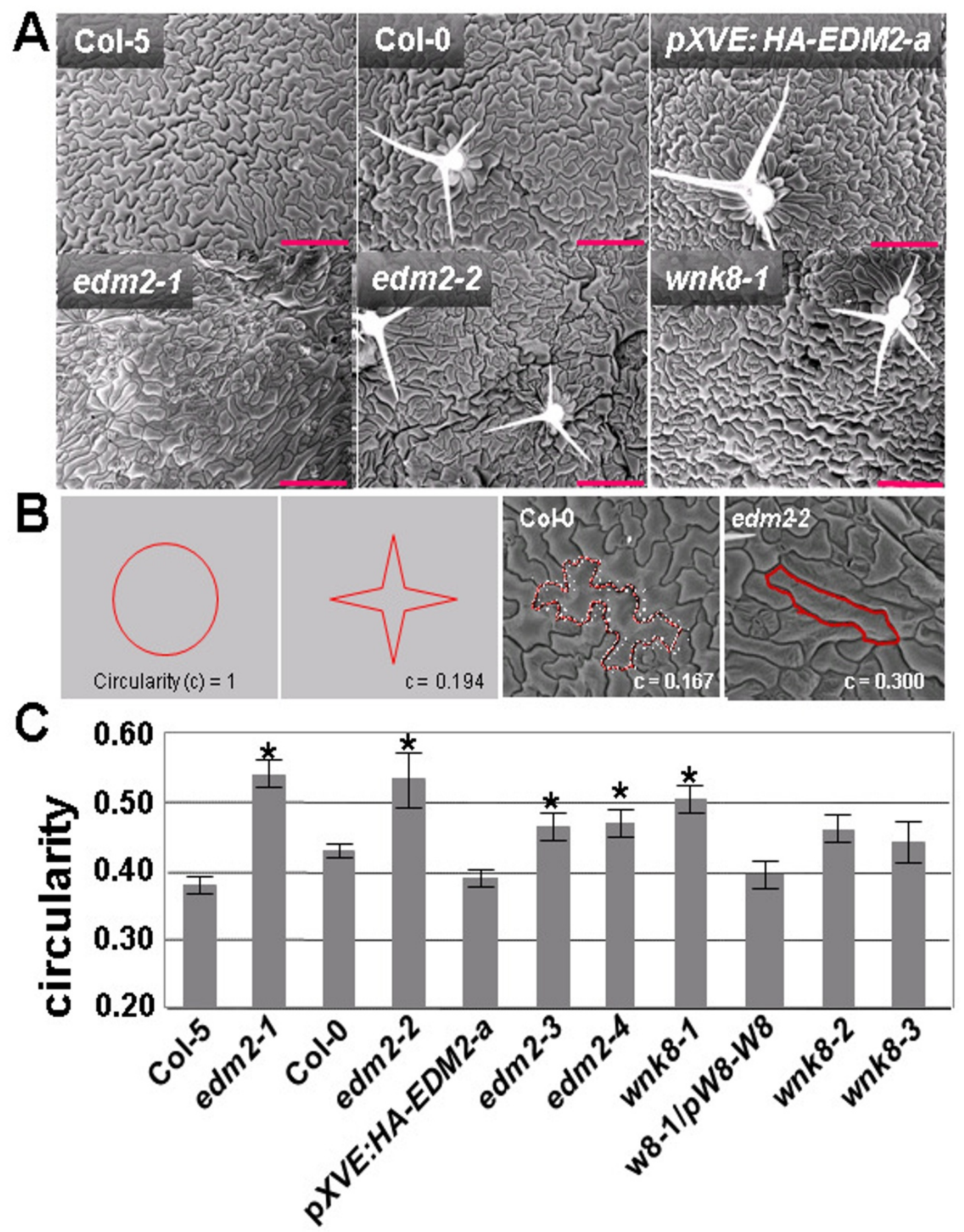

Figure 2 EDM2 and WNK8 are required for leaf pavement cell development. A: Scanning electron microscopy with adaxial surfaces of the 6th rosette leaves of the indicated Arabidopsis genotypes. 25 day-old plants grown under long-day conditions were used. The red scale bar represents $200 \mu \mathrm{m}$. pXVE:HA-EDM2-a plants were not treated with estradiol. B: Circularity [c $=4 \mathrm{pi} \times$ (area/perimeter $\left.\left.{ }^{2}\right)\right]$ as a numerical expression of cell shape. C: Average circularity values of 40 - 80 cells per genotype determined from the photos shown in (A) using Image $\mathrm{http}: / /$ rsbweb.nih.gov/ij/. Significant deviations of values from the tested mutants to their respective wild type controls (Col-5 for edm2-1, Col-0 for all others) based on $t$-tests $(p<0.05)$ are denoted by asterisks. 


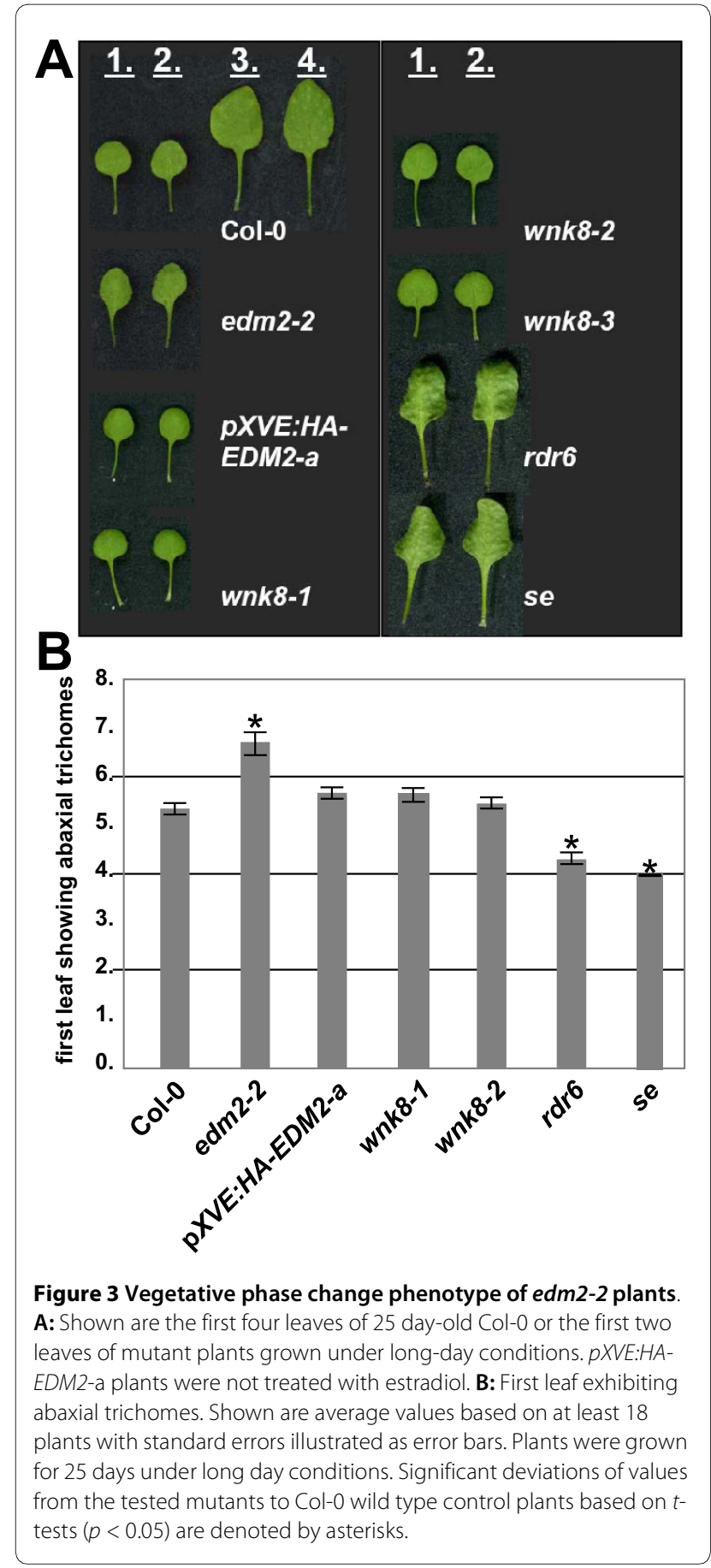

In Col-0 wild type plants, the onset of abaxial trichome production occurred in leaf 5 or 6 , whereas this phase transition was clearly delayed in edm2-2 plants (Figure $3 \mathrm{~B})$. In contrast, the development of leaves with abaxial trichomes was accelerated in the known phase change mutants $r d r 6$ and se (Figure $3 \mathrm{~B}$ ). Thus vegetative phase change seems rather to be delayed in $e d m 2-2$ and EDM2 appears to have a promoting role in this developmental transition. The morphological appearance of early edm2 leaves, which suggested the opposite, may not be related to vegetative phase change and may reflect the role of EDM2 in leaf pavement cell development (Figure 2). Both, the morphological phenotype of early edm2 leaves and the delayed development of abaxial trichomes are complemented in $p X V E: H A-E D M 2-a$ plants (Figure 3). None of the three $w n k 8$ mutant alleles or three independent WNK8 overexpressor lines, which exhibited clear flowering time phenotypes [2], showed any alterations of the leaf morphology and the vegetative phase transition (Figure 3 and not shown).

In summary, EDM2 is involved in multiple processes that affect distinct aspects of vegetative development, such as vegetative growth, the shape of leaf epidermis cells, and the phase of vegetative leaves. In each case, EDM2 acts as a positive regulator promoting the respective wild type processes. Furthermore, our data suggest that EDM2 and WNK8 act together in mechanisms promoting vegetative growth and epidermal cell development in leaves. As we did not observe any effect of $w n k 8$ mutants or WNK8 overexpressor lines related to vegetative phase change (this study) or EDM2/RPP7 mediated pathogen resistance [2], this protein kinase seems only to contribute to a subset of EDM2 functions.

\section{EDM2 and WNK8 have overlapping and co-directional effects on the transcriptome}

To assess interactions between EDM2 and WNK8 at the transcriptome level, we profiled transcript patterns of untreated Col-0, edm2-2, pXVE:HA-EDM2-a, wnk8-1, $w n k 8-2$, and $w n k 8-3$ seedlings using Affymetrix ATH1 whole genome arrays. For each genotype, signal intensities from three independent biological replicates were averaged and genes identified that were differentially expressed in the $e d m 2$ or $w n k 8$ mutants compared to either Col-0 or $p X V E: H A-E D M 2-a$ (see methods). Based on these data, we defined six primary sets of EDM2 or WNK8 target genes (Table 1; gene sets 1-6; Additional Files 1, 2, 3, 4, 5, 6) and derived six additional secondary sets of their targets (Table 1; gene sets 7 - 12; Additional Files $7,8,9,10,11,12$ ). Gene sets 1 and 2 as well as the secondary gene sets are also represented in Figure 4 . The sets "EDM2 induced" and "EDM2 suppressed" (sets 1 and 2) include genes whose transcript levels in edm2-2 compared to Col-0 are significantly lower or higher, respectively. The 222 genes of these two sets represent all genes we found to be differentially affected by EDM2. The more stringently defined sets "core EDM2 induced" and "core EDM2 suppressed" (Table 1; sets 3 and 4) are limited to those genes whose transcript levels, in addition to being differential in edm2-2, are reversed to wild type levels in $p X V E: H A-E D M 2-a$ plants. As expected, the EDM2 target gene $F L C$ [2] is included in the sets "EDM2 suppressed" 
Table 1: Sets of genes found to be differentially expressed in edm2 or wnk8 mutants by microarray experiments

\begin{tabular}{|c|c|c|c|}
\hline$\#$ & gene set name* & Definition** $^{* *}$ & number of genes*** \\
\hline 1 & EDM2-induced & Col-0 $>$ edm2-2 & 116 \\
\hline 2 & EDM2-suppressed & edm2-2 > Col-0 & 106 \\
\hline 3 & Core EDM2-induced & $\begin{array}{l}\text { Col-0 >edm2-2 AND } \\
\text { pXVE:HA-EDM2- } a>\text { edm2-2 }\end{array}$ & 9 \\
\hline 4 & core EDM2-suppressed & $\begin{array}{l}\text { edm2-2 > Col-0 AND } \\
\text { edm2-2 >pXVE:HA-EDM2- } a\end{array}$ & 37 \\
\hline 5 & WNK8-induced & $\begin{array}{l}\text { Col- } 0>w n k 8-1 \text { AND } \\
\text { Col-0 }>\text { wnk8-2 AND } \\
\text { Col-0 }>\text { }>\text { nnk-3 }\end{array}$ & 302 \\
\hline 6 & WNK8-suppressed & $\begin{array}{l}w n k 8-1>\text { Col-0 AND } \\
w n k 8-2>\text { Col-0 AND } \\
w n k 8-3>\text { Col-0 }\end{array}$ & 88 \\
\hline 7 & EDM2 \&WNK8-induced & set 1 AND set 5 & 66 \\
\hline 8 & EDM2 \&WNK8-suppressed & set2 AND set 6 & 15 \\
\hline 9 & only EDM2-induced & set1 NOT set5 & 50 \\
\hline 10 & only EDM2-suppressed & set2 NOT set 6 & 91 \\
\hline 11 & only WNK8-induced & set5 NOT set1 & 236 \\
\hline 12 & only WNK8-suppressed & set6 NOT set 2 & 73 \\
\hline
\end{tabular}

and "core EDM2 suppressed". RPP7 is not represented on the ATH1 array.

"WNK8 induced" and "WNK8 suppressed" (Table 1; sets 5 and 6) represent genes whose transcript levels in each of the three wnk 8 mutants compared to Col- 0 are significantly lower or higher, respectively. The 390 genes of these two sets represent all genes we found to be consistently expressed differentially in all three wnk8 mutants. Although this complement of WNK8 target genes (which is based on altered transcript levels in the three mutants) is more stringently defined than the complement of the EDM2 target genes (which is only based on altered transcript levels in one mutant), the former is almost twice as large as the latter, suggesting that WNK8 may have a wider spectrum of biological roles than EDM2. More than a third (36.5\%) of the 222 genes differentially regulated by EDM2 ("EDM2 induced" or "EDM2 suppressed") are also differentially regulated by WNK8 ("WNK8 induced" or "WNK8 suppressed"; Figure 4). Strikingly, all of these 81 genes commonly controlled by EDM2 and WNK8 are affected by these two regulators in the same manner, as 15 of them are EDM2 and WNK8 suppressed, while the remaining 66 are EDM2 and WNK8 induced (Table 1; gene sets $7 \& 8$ ). This strict co- 


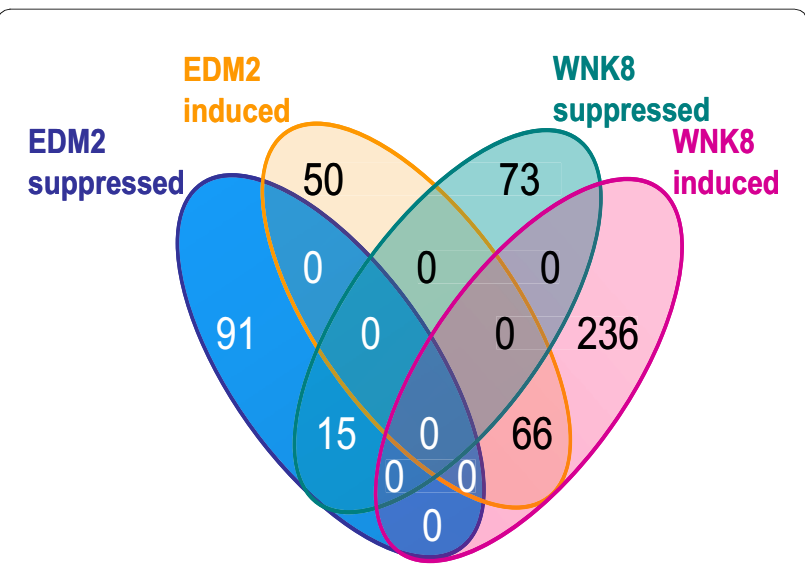

Figure 4 Venn diagram showing overlaps between the indicated sets of EDM2- or WNK8-dependent expressed genes.

directionality of EDM2 and WNK8-dependent transcriptional changes indicates a close functional connection between both regulators and is consistent with the physical interactions between them [2] as well as the fact that they affect a common set of biological processes.

In order to infer possible molecular and/or biological processes controlled commonly by EDM2 and WNK8 or exclusively by each of these two regulators individually we used the "AmiGO" program http://amigo.geneontology.org/cgi-bin/amigo/term_enrichment to identify overrepresented Gene Ontology (GO) terms in gene sets 7 12 (defined in Table 1). As shown in Table 2, EDM2 and WNK8 commonly promote expression of genes involved in metabolic processes as well as genes responsive to various stimuli, such as stress, temperature, chemicals and other abiotic cues (Table 2, set 7). Similar themes are found in the sets of genes exclusively and not commonly controlled by EDM2 and WNK8. While the set of "only EDM2-induced" genes is enriched for genes involved in catalytic activity (Table 2, set 9), the set of "only WNK8induced" genes includes genes responsive to a variety of stimuli, such as stress, temperature, chemicals and abiotic cues (Table 2, set 11). Thus, the roles of EDM2 and WNK8 appear to be partially independent of each other. However, both regulators seem to cooperate regarding a subset of their functions. Interestingly, EDM2 appears to suppress a small set of genes responsive to biotic stimuli and other organisms (Table 2, set 10). This set includes LURP (Late Upregulated in Response to Hpa)_1 and ACD (Accelerated Cell Death) 6, which we previously found to be $\mathrm{Hpa}$-inducible by an EDM2 dependent mechanism [1]. Hence, EDM2 seems to be involved in the Hparesponsive activation of a small set of defense genes as well as their suppression in the absence of Hpa.

\section{Discussion}

This study together with data already published [2] show that the Arabidopsis defense regulator EDM2 has multiple additional roles in development. Its roles are diverse and besides innate immunity, include other apparently unrelated processes, such as development of leaf pavement cells, vegetative phase change as well as control of floral transition.

\section{EDM2 is involved in several regulatory modules}

EDM2 acts together with WNK8 in controlling the floral transition, while WNK8 appears not to have any role in EDM2/RPP7-mediated disease resistance to HpaHiks1 [2]. Thus, an EDM2/WNK8-dependent mechanism controls flowering time while a second EDM2-dependent mechanism that seems not to involve WNK8 mediates resistance to HpaHiks1. Related to the latter regulatory circuit may be the vegetative phase change-related function of EDM2, which is also affected in edm2 mutants but not in $w n k 8$ mutants or WNK8 overexpressor lines. Other roles of EDM2, such as those in vegetative growth and development of leaf epidermal cells may represent a third category of EDM2-dependent regulatory mechanisms, as they seem to involve WNK8. In contrast to the floral transition, however, which is promoted by EDM2 and counteracted by WNK8, vegetative growth and epidermal cell morphology are affected in the same manner by EDM2 and WNK8, which both positively contribute to these developmental processes. Thus, at least three distinct EDM2-dependent regulatory modules that differ in their dependency on and interactions with WNK8 can be discriminated (Figure 5).

\section{Effects of EDM2 and WNK8 on leaf pavement cell morphology and vegetative growth}

Both EDM2 and WNK8 positively contribute to proper development of leaf pavement cells as well as overall vegetative growth. In each case, mutations in EDM2 have a stronger impact on the respective phenotype than mutations in WNK8. Despite having qualitatively similar effects on leaf pavement cells and vegetative growth, we cannot exclude that EDM2 and WNK8 independently contribute to these developmental processes. Fu et al. [6] found the "jigsaw-like" appearance of Arabidopsis leaf pavement cells to result from the coordinated interplay of the small Rho-type GTPases ROP2 (Rho-related GTPases from plants 2) and ROP4 and their interacting partners RIC1 (ROP-interactive CRIB motif-containing protein 1) and RIC4. In addition, ANGUSTIFOLIA has been found to mediate the formation of protrusions in Arabidopsis leaf cells [14]. However, transcript levels of these developmental regulators appear not to be altered by mutations in EDM2 or WNK8 in our microarray experiments. Thus, EDM2 and WNK8 may either affect transcript levels of 
Table 2: Overrepresented GO attributes in sets of differentially expressed genes

\begin{tabular}{|c|c|c|c|}
\hline Gene set* & $\begin{array}{l}\text { GO term } \\
\text { (and category)** }\end{array}$ & p-value & Genes*** \\
\hline \multirow[t]{9}{*}{ set 7 "EDM2 \&WNK8-induced" } & metabolic process (BF) & $\begin{array}{l}3.08 \\
e-05\end{array}$ & $\begin{array}{l}\text { INOSITOL(1,4,5)P3 5-PHOSPHATASE II, CKX4, XTH18, } \\
\text { CYP86A4, CH1, AT5G42250, AT5G25930, GPAT3, } \\
\text { MBF1C, CRK10, AKN2, ATCAD4, JAR1, SHM7, } \\
\text { AT5G45650, CYP81F2, WRKY40, ATGA2OX2, } \\
\text { AT2G05940, OPR3, AT5G37540, ATHSP101, BMY3, } \\
\text { SCL13, GSTU12, APS3, PUB23, AT3G04010, NUDT7, } \\
\text { AT2G32150, ATGSTU5, XTR6, AT1G70740, CRK42, } \\
\text { GPAT2, RBOHD, AT1G11050, KCS9, ACA1, SZF1 }\end{array}$ \\
\hline & response to stimulus (BF) & $\begin{array}{l}3.01 \\
e-04\end{array}$ & $\begin{array}{l}\text { INOSITOL(1,4,5)P3 5-PHOSPHATASE II, WRR4, } \\
\text { AT4G34150, MBF1C, AT1G59860, JAR1, SHM7, } \\
\text { CYP81F2, WRKY40, ATGA2OX2, OPR3, ATHSFA2, } \\
\text { ATHSP101, SCL13, CAD1, PUB23, NUDT7, ATGSTU5, } \\
\text { TCH4, AT3G09440, RBOHD, KCS9, SZF1 }\end{array}$ \\
\hline & $\begin{array}{l}\text { response to temperature } \\
\text { stimulus (BF) }\end{array}$ & $\begin{array}{l}9.73 \\
\mathrm{e}-04\end{array}$ & $\begin{array}{l}\text { AT4G34150, MBF1C, AT1G59860, ATHSFA2, } \\
\text { ATHSP101, TCH4, RBOHD, KCS9 }\end{array}$ \\
\hline & response to stress (BF) & $\begin{array}{l}2.10 \\
e-03\end{array}$ & $\begin{array}{l}\text { WRR4, AT4G34150, MBF1C, AT1G59860, JAR1, } \\
\text { CYP81F2, WRKY40, OPR3, ATHSFA2, ATHSP101, } \\
\text { PUB23, NUDT7, ATGSTU5, TCH4, RBOHD, KCS9 }\end{array}$ \\
\hline & $\begin{array}{l}\text { response to chemical stimulus } \\
\text { (BF) }\end{array}$ & $\begin{array}{l}3.98 \\
\mathrm{e}-03\end{array}$ & $\begin{array}{l}\text { INOSITOL(1,4,5)P3, 5-PHOSPHATASE, II, MBF1C, } \\
\text { JAR1, SHM7, WRKY40, OPR3, ATHSFA2, ATHSP101, } \\
\text { SCL13, PUB23, NUDT7, ATGSTU5, TCH4, AT3G09440, } \\
\text { SZF1 }\end{array}$ \\
\hline & cellular metabolic process (BF) & $\begin{array}{l}5.01 \\
e-03\end{array}$ & $\begin{array}{l}\text { INOSITOL(1,4,5)P3 5-PHOSPHATASE II, CKX4, XTH18, } \\
\text { CYP86A4, CH1, AT5G25930, MBF1C, CRK10, AKN2, } \\
\text { ATCAD4, JAR1, SHM7, CYP81F2, WRKY40, } \\
\text { ATGA2OX2, AT2G05940, OPR3, ATHSP101, SCL13, } \\
\text { GSTU12, APS3, PUB23, NUDT7, ATGSTU5, XTR6, } \\
\text { AT1G70740, CRK42, RBOHD, AT1G11050, KCS9, } \\
\text { ACA1, SZF1 }\end{array}$ \\
\hline & $\begin{array}{l}\text { response to abiotic stimulus } \\
\text { (BF) }\end{array}$ & $\begin{array}{l}7.64 \\
e-03\end{array}$ & $\begin{array}{l}\text { AT4G34150, MBF1C, AT1G59860, JAR1, ATGA2OX2, } \\
\text { ATHSFA2, ATHSP101, PUB23, NUDT7, TCH4, RBOHD, } \\
\text { KCS9 }\end{array}$ \\
\hline & catalytic activity (MF) & $\begin{array}{l}3.57 \\
e-09\end{array}$ & $\begin{array}{l}\text { INOSITOL(1,4,5)P3 5-PHOSPHATASE II, CKX4, XTH18, } \\
\text { CYP86A4, CH1, WRR4, AT5G42250, AT4G24160, } \\
\text { GPAT3, CRK10, AKN2, ATCAD4, AT1G72520, JAR1, } \\
\text { SHM7, AT5G45650, AT5G65140.2, CYP81F2, } \\
\text { ATGA2OX2, AT2G05940, OPR3, AT5G37540, } \\
\text { ATHSP101, AT1G21120, BMY3, AT5G39580, GSTU12, } \\
\text { APS3, PUB23, AT3G04010, SUB, NUDT7, AT2G32150, } \\
\text { ATGSTU5, XTR6, TCH4, AT1G70740, AT1G73600, } \\
\text { CRK42, GPAT2, RBOHD, KCS9, ACA1 }\end{array}$ \\
\hline & transferase activity (MF) & $\begin{array}{l}7.87 \\
\mathrm{e}-05\end{array}$ & $\begin{array}{l}\text { XTH18, AT4G24160, GPAT3, CRK10, AKN2, JAR1, } \\
\text { SHM7, AT2G05940, AT1G21120.1, GSTU12, APS3, } \\
\text { SUB, ATGSTU5, XTR6, TCH4, AT1G70740.1, } \\
\text { AT1G73600, CRK42, GPAT2, KCS9 }\end{array}$ \\
\hline
\end{tabular}




\section{Table 2: Overrepresented GO attributes in sets of differentially expressed genes (Continued)}

\begin{tabular}{lll}
\hline set 9 & catalytic activity (MF) & AT5G39080, AT3G63510, AAE14, PSD2, AT1G22430, \\
"only EDM2-induced" & SAL1, ATALN, AT5G09300, AT4G31390, AT2G25870, \\
& SRS, FC2, AT2G47880, AtCXE17, CSLA11, PKp3, \\
& UGT80B1, MCM4, ADC1, AT5G61480, AT2G26870 \\
& AT4G33920, CYP78A8, B120, XCP1, ATPREP2, \\
& CYP79B2, AT2G04845, AT5G24670, PAL4, CINV2,
\end{tabular}

\begin{tabular}{|c|c|c|c|}
\hline $\begin{array}{l}\text { set } 10 \\
\text { "only EDM2-suppressed" }\end{array}$ & $\begin{array}{l}\text { response to biotic stimulus } \\
\text { (BF) }\end{array}$ & $\begin{array}{l}1.84 \\
e-03\end{array}$ & $\begin{array}{l}\text { PCC1, ATCNGC11, ATEBP, FER1, AZI1, PR4, ACD6, } \\
\text { pEARLI, 1, PR5, LURP1, MLP34 }\end{array}$ \\
\hline & $\begin{array}{l}\text { response to other organism } \\
\text { (BF) }\end{array}$ & $\begin{array}{l}8.71 \\
e-03\end{array}$ & $\begin{array}{l}\text { PCC1, ATCNGC11, ATEBP, FER1, AZI1, PR4, ACD6, } \\
\text { pEARLI, 1, PR5, LURP1 }\end{array}$ \\
\hline $\begin{array}{l}\text { set } 11 \\
\text { "only WNK8-induced" }\end{array}$ & Response to stimulus (BF) & $\begin{array}{l}6.23 \\
e-15\end{array}$ & $\begin{array}{l}\text { AT3G42570, GPT2, TIL, STO, CBP60G, BAM1, } \\
\text { AT1G51090, AT3G61220, HSP17.6II, OPR2, WRKY18, } \\
\text { LOX3, AT2G29500, VTC4, ERD10, AT3G53990, } \\
\text { MYB77, CRK11, TCH3, AT1G03220.1, PIL6, CIPK20, } \\
\text { WRKY25, WRKY33, BT5, ZAR1, AT3G04210.1, } \\
\text { ATHSP90.1, MEK1, ATHB-12, AT5G50915, HSP70, } \\
\text { MYBR1, EXO, HSP17.4, LZF1, ATCAMBP25, CMPG1, } \\
\text { PHT4;1, AT-HSP17.6A, ATPAP1, ATACA2, SYP122, } \\
\text { PAPP2C, LHCB2.3, SOUL-1, TCH2, NHL2, CPK32, } \\
\text { CYP707A3, ARR6, SFP1, RAV1, DIN11, AT4G28140, } \\
\text { UTR1, LEA14, AT1G55450.1, CEJ1, ATGSTU26, } \\
\text { AT1G54050, JAZ6, TIR, NIR1, ADR1, NHL3, } \\
\text { AT5G51440, SYP121, TMAC2, CML38, AT5G51190, } \\
\text { AT5G54170, ATGSTF8, AT1G19020, ADOF1, CAM9 }\end{array}$ \\
\hline & response to stress (BF) & $\begin{array}{l}1.26 \\
\mathrm{e}-13\end{array}$ & $\begin{array}{l}\text { AT3G42570, TIL, STO, CBP60G, BAM1, AT1G51090, } \\
\text { AT3G61220, HSP17.6Il, OPR2, WRKY18, LOX3, } \\
\text { AT2G29500, VTC4, ERD10, AT3G53990, CRK11, } \\
\text { AT1G03220, WRKY25, WRKY33, ZAR1, AT3G04210, } \\
\text { ATHSP90.1, MEK1, ATHB-12, HSP70, MYBR1, } \\
\text { HSP17.4, ATCAMBP25, AT-HSP17.6A, ATPAP1, } \\
\text { SYP122, TCH2, NHL2, CPK32, CYP707A3, DIN11, } \\
\text { UTR1, LEA14, AT1G55450, CEJ1, ATGSTU26, } \\
\text { AT1G54050, JAZ6, TIR, ADR1, NHL3, AT5G51440, } \\
\text { SYP121, TMAC2, CML38, AT5G54170, ATGSTF8, } \\
\text { AT1G19020, CAM9 }\end{array}$ \\
\hline
\end{tabular}

response to abiotic stimulus $\quad 2.47$

(BF)

e-10
TIL, STO, BAM1, HSP17.6II, AT2G29500, VTC4, ERD10, AT3G53990, TCH3, AT1G03220.1, PIL6, WRKY25, WRKY33, ATHSP90.1, MEK1, ATHB-12, HSP70, MYBR1, HSP17.4, LZF1, ATCAMBP25, ATHSP17.6A, PAPP2C, LHCB2.3, SOUL-1, TCH2, CPK32, CYP707A3, LEA14, AT1G55450.1, CEJ1, ATGSTU26, AT1G54050, ADR1, AT5G51440, TMAC2, ATGSTF8, CAM9

AT3G42570, GPT2, CBP60G, BAM1, WRKY18, AT2G29500, ERD10, MYB77, CRK11, CIPK20, WRKY33, BT5, ATHSP90.1, MEK1, ATHB-12, AT5G50915, HSP70, MYBR1, EXO, ATCAMBP25, CMPG1, AT-HSP17.6A, ATACA2, SYP122, TCH2, CPK32, CYP707A3, ARR6, RAV1, AT4G28140, UTR1, LEA14, CEJ1, ATGSTU26, AT1G54050, JAZ6, NIR1, ADR1, SYP121, TMAC2, AT5G51190, AT1G19020, ADOF1, CAM9 
Table 2: Overrepresented GO attributes in sets of differentially expressed genes (Continued)

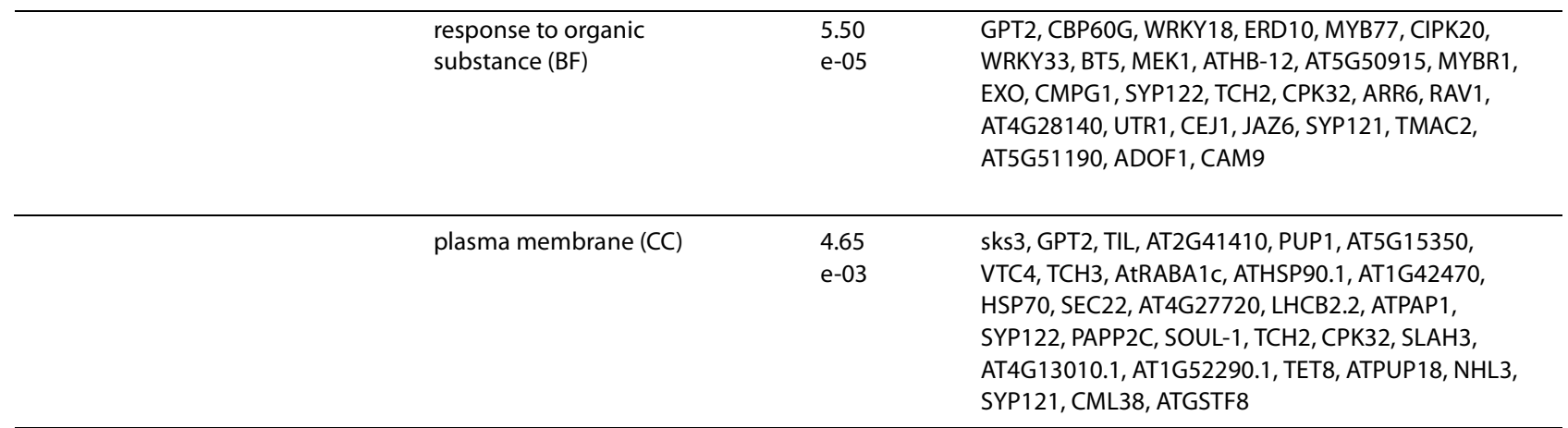

*: Gene sets 7 - 12, as defined in table 1 were examined for overrepresented GO attributes using AmiGO. ${ }^{* *}$ : GO terms belonging to either of the three GO categories "Biological Function" (BF), "Molecular Function" (MF) or "Cellular Compartment" (CC) are listed. ***: If available common gene names are listed; otherwise AGI gene IDs are given.

other components of the respective regulatory pathways or affect them by playing roles other than transcriptional regulation.

Besides changes of epidermal cell morphology, mutations in EDM2 also impact the overall size and morphol-

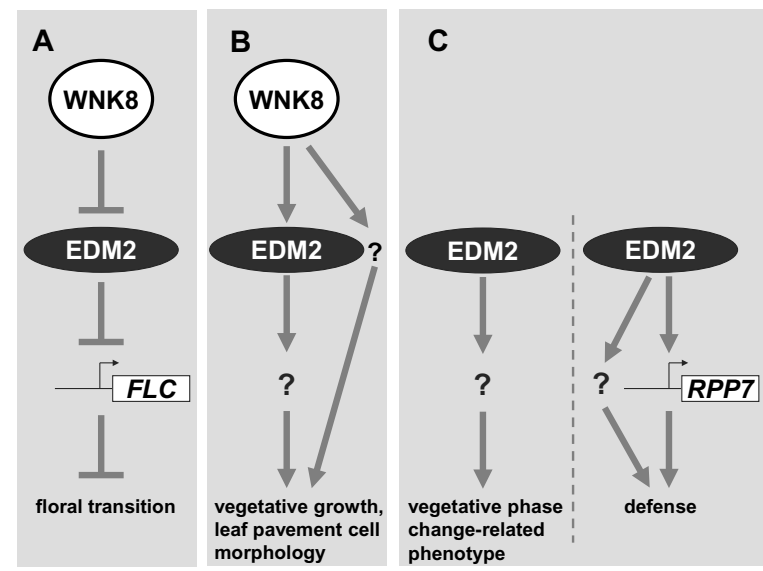

Figure 5 Different types of EDM2-dependent regulatory modules. Based on differential effects of EDM2 and WNK8 on developmental or defense processes, three types of EDM2-dependent regulatory modules can be discriminated. A: WNK8/EDM2-dependent control of flowering time. Genetic analyses showed that WNK8 operates upstream from EDM2 counteracting the suppressing effect of EDM2 on FLC transcript levels. B: WNK8/EDM2-dependent control of vegetative growth and epidermal cell morphology. Both WNK8 and EDM2 promote these developmental processes. WNK8 may signal via EDM2 or in parallel to EDM2. C: EDM2-dependent control of early vegetative phase change and RPP7- dependent disease resistance to HpaHiks 1 . These processes are independent from WNK8. Disease resistance is likely mediated through regulation of RPP7transcript levels. Additional parallel steps may also contribute to this response. A, B, C: Arrows indicate promoting effects, while "?" symbols indicate suppressing effects. ogy of rosette leaves, which are less expanded in edm2 plants compared to Col-0 resulting in a reduced fresh weight of these mutants. This phenotype may be a consequence of the altered leaf pavement cell shape. Several examples indicate causal links between abnormal development of leaf cell morphology and leaf organogenesis [15] and mutations in ANGUSTIFOLIA or ROP2 also result in more narrow rosette leaves $[16,17]$. The reduction of leaf size and fresh weight in wnk8 mutants is less pronounced than in $e d m 2$ mutants. Consistent with this, their leaf pavement cell phenotype is also less pronounced compared to $e d m 2$ mutants.

\section{Vegetative phase change-related function of EDM2}

While RPP7-mediated resistance to HpaHiks1 is strongly dependent on EDM2 [1], this immune response appears not to require WNK8. Similarly, the vegetative phase change-related phenotype of $e d m 2$ mutants appears to be absent in any of the $w n k 8$ mutants or WNK 8 overexpressor lines. Thus, the roles of EDM2 in RPP7-mediated resistance and vegetative phase change appear to be distinct from its roles in the control of flowering time, leaf pavement cell development and vegetative growth. A pathway for the biogenesis of trans-acting siRNAs involving HASTY, ZIPPY, SGS3 and RDR6 has been implicated in the control of early vegetative phase change in Arabidopsis. However, neither transcript levels of these genes nor of five genes that seem to be controlled by this pathway (ARF3 [AUXIN RESPONSE FACTOR 3; At2g33860], ARF4 [AUXIN RESPONSE FACTOR 4, At5g60450], SPL3 [SQUAMOSA PROMOTER BINDING PROTEIN-LIKE 3, At2g33810], At1g63130 and At5g18040) [12] appear to be affected by EDM2 in our microarray experiments. Thus, involvement of EDM2 in this siRNA pathway seems unlikely. 


\section{EDM2/WNK8-dependent regulation of floral transition}

We previously found EDM2 and WNK8 to operate in a regulatory module controlling flowering time [2]. EDM2 has a promoting effect on this developmental transition, while WNK8 appears to counteract this EDM2 function. Given their antagonistic effects on the floral transition, the co-directionality of EDM2 and WNK8-dependent transcriptome changes seems counter-intuitive. However, our microarray analyses were performed with vegetative tissues prior to the floral induction. Thus, transcriptional effects caused by EDM2- or WNK8-modulated FLC activity should not be represented in our microarray data set.

As we describe previously [2], the role of EDM2 in promoting the floral transition by suppressing the floral repressor $F L C$ in a photoperiod-independent manner resembles that of the well-characterized autonomous floral promotion pathway [3]. So far seven regulatory genes have been associated with this pathway, FCA (At4g16280), FY (At5g13480), FLK (FLOWERING LOCUS KH DOMAIN, At3g04610), FPA (At2g43410), FLD (FLOWERING LOCUS D, At3g10390), FVE (At2g19520) and LD (LUMINIDEPENDENS, AT4G02560) [18-21]. We did not observe in our microarray experiments differential effects on the transcript levels of any autonomous pathway gene in edm2-2 ruling out that EDM2 affects this pathway simply by controlling the expression of one of its known components. A role of EDM2 as a member of the autonomous pathway is further supported by our microarray data, which revealed substantial overlap between transcriptional signatures of the fpa/fld double mutant [3] as well as the edm2 and wnk8 mutants (Additional File 13, Figure S1). The fact that autonomous pathway mutants (like edm2 mutants) exhibit delayed vegetative phase change is also consistent with the proposed function of EDM2 as a new component of this floral promotion pathway [13].

\section{Co-option of EDM2 to distinct regulatory processes}

According to whole genome sequences available from Genbank http://blast.ncbi.nlm.nih.gov/Blast.cgi, EDM2like proteins (ELPs, as defined by [1]) appear to have originally evolved from a common ancestor of land plants, as they are present in eudicots, monocots and bryophytes, but are lacking in prokayotes, green algae, fungi, protozoans or metazoans. While key mechanisms of plant development must have emerged early in the lineage of land plants, the acquisition of specific mechanisms of pathogen recognition and defense activation are likely more recent events resulting from co-evolution of higher plants along with pathogens adapting to their hosts [22]. Thus, we believe that at least some of the developmental roles of EDM2 reflect more ancient functions than its role in defense. Thus, EDM2 as a regulator of some developmen- tal processes may have been co-opted to control additional functions including defense. Co-option, the recruitment of extant genes to new functions [23], often involves the utilization of new promoter elements leading to a wider spectrum of expression and, consequently, deployment of the respective gene products into new regulatory or metabolic contexts. Alternatively, new domains can be added to an extant protein enabling it to execute new molecular roles in addition to its ancestral functions. Of interest in this respect may be the C-terminal extension of EDM2, which we found to act as a potent transcriptional activation domain (T.T. \& T.E. unpublished). This C-terminal extension is only present in ELP family members of the eudicot clade [1]. Thus, the relatively recent acquisition of the $\mathrm{C}$-terminal extension may be responsible for a widened functional spectrum of EDM2. Future studies will have to address if EDM2 orthologs that lack this domain have a reduced set of EDM2-related roles.

At least some EDM2 functions must be occurring simultaneously in the same cell types. EDM2 and WNK8 transcripts are detectable in all aerial vegetative tissues as well as in the shoot apex during and after the floral transition (AtGenExpress; http://jsp.weigelworld.org/expviz/ expviz.jsp). As most vegetative aerial Arabidopsis tissues appear to be capable of defense responses to Hpa [24], roles of EDM2 in immunity are likely to spatially coincide with its roles in vegetative phase change or leaf pavement cell development and vegetative growth. Co-option of EDM2 to multiple simultaneous functions may reflect an important adaptation allowing for crosstalk between apparently unrelated biological processes. For example, limited amounts of cellular EDM2 protein may constitute a "regulatory bottleneck" enabling the plant to balance the allocation of its resources between defense responses and different developmental processes.

Additionally, as discussed elsewhere [2], EDM2-dependent developmental processes may be manipulated by biotrophic pathogens, such as HpaHiks1. A typical feature of pathogens that are well adapted to their hosts is the use of effector molecules, which they secrete into host cells to increase pathogen virulence. Such effectors or virulence factors can attenuate host defense processes or change plant metabolism or development [25] in order to generate a more favorable environment for the pathogen in the host. Often plants have evolved mechanisms to recognize effector-mediated interferences with their regulatory apparatus to activate strong immune responses $[26,27]$. A similar scenario may explain the involvement of EDM2 in developmental and immune processes.

\section{Conclusions}

Besides RPP7-mediated immunity, EDM2 positively affects several developmental processes which include 
the formation of leaf pavement cells, leaf expansion, a role related to vegetative phase change as well as floral transition. These EDM2-dependent processes differ with respect to their dependency on the EDM2-interating protein kinase WNK8. While WNK8 seems not to affect the vegetative phase change-related role of EDM2, it has a promoting effect on the development of leaf pavement cells and leaf expansion. The fact that the effects of EDM2 and WNK8 on leaf pavement cell formation and leaf expansion are co-directional, while WNK8 counteracts the promoting effect of EDM2 on the floral transition suggests that WNK8 can modulate the activity of EDM2. Thus, we propose that EDM2 has been co-opted to distinct regulatory modules controlling a set of different processes in plant immunity and development. Some functions of these EDM2 functions appear to be modulated by WNK8.

\section{Methods \\ Mutant lines}

Wild-type plants are the Arabidopsis accession Col-0 and Col-5 which were obtained from the Arabidopsis Biological Resource Center (ABRC). The edm2-1, edm2-2, edm2-3 and edm2-4, pXVE:HA-EDM2- $a$, wnk8-1, wnk82, wnk8-3, rdr6 (sgs2-1) and se mutant lines were described previously [1,2,4,28,29]. Briefly: The edm2-1 mutant contains a fast neutron-induced deletion of $2 \mathrm{bp}$ creating a premature stop codon predicted to result in a truncated protein product. The edm2-2, edm2-3 and edm2-4 SALK T-DNA lines contain insertions in the $4^{\text {th }}$ and $5^{\text {th }}$ of 17 introns (edm2-2 \&edm2-3, respectively) or the $9^{\text {th }}$ of 18 exons (edm2-4). While $e d m 2-2$ appears to be an mRNA null allele, low transcript levels of $e d m 2-1$ are detectable. Transcript levels of $e d m 2-3$ and $e d m 2-4$ are partially reduced (T.T. \& T.E. unpublished). The $w n k 8-1$ and wnk8-2 SALK T-DNA mutants contain insertions in the $5^{\text {th }}$ and $7^{\text {th }}$ of seven exons, respectively, resulting in reduced levels of WNK8 transcript. The wnk8-3 GABIKat T-DNA mutant harbors an insertion in the $2^{\text {nd }}$ of six introns resulting in strongly reduced WNK8 transcript levels. The se mutant was generated by $\mathrm{X}$-ray mutagenesis of Col-1 plants and contains a 7 bp deletion causing a frameshift altering the last 27 amino acids of the predicted Serrate protein.

\section{Transgenic lines}

For the functional complementation of the wnk8-1 mutation, genomic DNA fragments containing the WNK8 gene as well as its entire upstream intergenic region were PCR-amplified using primers, gWNK8GW-F (5'GGGGACAAGTTTGTACAAAAAAGCAGGCTGCTCTTACGTACATCGTCTTTTTCAC-3') gWNK8GW-R GGGGACCACTTTGTACAAGAAAGCTGGGTAT-
CAAGAGATGTTAACTGCTTTTTGC-3'). The amplified genomic DNA fragment was introduced into pDONR/Zeo plasmid (Invitrogen) to generate a Gateway entry clone and the cloned WNK8 genomic fragment was transferred into the pEarleyGate302 vector [30]. the resulting construct was transformed into wnk8-1.

\section{Microscopy}

In order to observe the shape of leaf epidermal pavament cells, scanning electron microscopy was performed with fresh $6^{\text {th }}$ rosetta leaves of wild type or mutant lines using a Hitachi TM-1000 scanning electron microscope (Hitachi High-Technologies). Individual images were processed using Image J software http://rsb.info.nih.gov/ij/ to measure circularity. The appearance of abaxial trichomes was monitored using a stereomicroscope.

\section{Microarray analysis}

Arabidopsis plants were grown on soil under short day conditions ( $8 \mathrm{~h}$ light $/ 16 \mathrm{~h}$ dark, $22^{\circ} \mathrm{C}$ and light intensity of 120 umol m-2 $\mathrm{s}^{-1}$ ). 2-week-old seedlings were harvested 7 to 8 hour after dawn and total RNA was isolated using TRIzol Reagenet (Invitrogen). RNA was further purified with RNeasy Plant mini kit columns (Qiagen) according to the protocols supplied by the manufacturers. The purified RNA was processed and hybridized to the Affymetrix Arabidopsis ATH1 genome array GeneChip following the manufacturer's instructions (Affymetrix) by the IIGB Core Instrumentation Facility of University of California at Riverside. Three independent biological replicates were performed for each treatment. Data was processed in the statistical programming environment $R$ using Bioconductor packages. Raw expression values were normalized using the robust multichip averaging algorithm. Analysis of differentially expressed genes (DEGs) was performed with the LIMMA package [31]. The Benjamini and Hochberg method was selected to adjust $P$ values for multiple testing [32]. Differentially expressed genes were ranked using the B statistics, and these exhibiting a B-statistic of greater than 0 were considered differentially expressed. Venn diagrams were constructed using the Venny tool http:// bioinfogp.cnb.csic.es/tools/venny/index.html. The AmiGO program http://amigo.geneontology.org/cgi-bin/ amigo/term enrichment was applied to identify enriched Gene Ontology (GO) terms in sets of co-expressed genes. The following AmiGO program settings were used: "Database Filter": "TAIR"; "Maximum p-value": "0.01"; Minimum number of gene products": "10\% of input gene set".

\section{Accession Numbers}

(5'- Sequence data from this article can be found in the Arabidopsis Genome Initiative or GenBank/EMBL databases 
under the following accession numbers: EDM2 (AT5G55390), WNK8 (AT5G41990). The microarray data have been deposited in MIAME-compliant format in the GEO database under the accession number GSE17499.

\section{Additional material}

Additional file 1 Table S1: Microarray Data Set 1. List of genes in microarray data set 1 (as defined in Table 1).

Additional file 2 Table S2: Microarray Data Set 2. List of genes in microarray data set 2 (as defined in Table 1).

Additional file 3 Table S3: Microarray Data Set 3. List of genes in microarray data set 3 (as defined in Table 1)

Additional file 4 Table S4: Microarray Data Set 4 . List of genes in microarray data set 4 (as defined in Table 1).

Additional file $\mathbf{5}$ Table S5: Microarray Data Set $\mathbf{5}$. List of genes in microarray data set 5 (as defined in Table 1).

Additional file 6 Table S6: Microarray Data Set 6 . List of genes in microarray data set 6 (as defined in Table 1).

Additional file 7 Table S7: Microarray Data Set 7. List of genes in microarray data set 7 (as defined in Table 1).

Additional file 8 Table S8: Microarray Data Set 8. List of genes in microarray data set 8 (as defined in Table 1).

Additional file $\mathbf{9}$ Table S9: Microarray Data Set $\mathbf{9}$. List of genes in microarray data set 9 (as defined in Table 1).

Additional file 10 Table S10: Microarray Data Set $\mathbf{1 0}$. List of genes in microarray data set 10 (as defined in Table 1).

Additional file 11 Table S11: Microarray Data Set 11. List of genes in microarray data set 11 (as defined in Table 1).

Additional file 12 Table S12: Microarray Data Set 12. List of genes in microarray data set 12 (as defined in Table 1).

Additional file 13 Figure S1: Venn diagrams. Venn diagrams representing overlapping sets of EDM2/WNK8 controlled genes and genes controlled by the autonomous pathway genes $f p a, f d$ and fld.

\section{Authors' contributions}

TT designed and performed all experiments and wrote parts of the manuscript. TE supervised this project and wrote parts of the manuscript. All authors read and approved the final manuscript.

\section{Acknowledgements}

We thank Drs. Patricia Springer, Harley Smith and Yu-Hung (Linda) Wei (all UCRiverside) for helpful discussions, technical advice and critical reading of the manuscript. We also thank Jonathan Weiz (UC-Riverside) for excellent technical assistance and the ABRC and NASC stock centers for providing Arabidopsis TDNA mutants. This work was supported by NSF grant IOS-0724838-003 to TE.

\section{Author Details}

Center for Plant Cell Biology, Institute for Integrative Genome Biology, Department of Botany and Plant Sciences, University of California at Riverside, Riverside, CA 92521, USA

Received: 15 March 2010 Accepted: 14 September 2010 Published: 14 September 2010

\section{References}

1. Eulgem T, Tsuchiya T, Wang X, Cuzick A, Beasley B, Toer M, McDowell JM, Holub E, Zhu T, Dangl JL: EDM2 is required for RPP7-dependent disease resistance in Arabidopsis and affects RPP7 transcript levels. The Plant Journal 2007, 49:829-839.

2. Tsuchiya T, Eulgem T: The Arabidopsis defense component EDM2 affects the floral transition in an FLC-dependent manner. The Plant Journal 2010, 62:518-528.

3. Veley KM, Michaels SD: Functional redundancy and new roles for genes of the autonomous floral-promotion pathway. Plant Physiol 2008, 147(2):682-695.
4. Wang Y, Liu K, Liao H, Zhuang C, Ma H, Yan X: The plant WNK gene family and regulation of flowering time in Arabidopsis. Plant Biol (Stuttg) 2008, 10(5):548-562.

5. Gavin AC, Bosche M, Krause R, Grandi P, Marzioch M, Bauer A, Schultz J, Rick JM, Michon AM, Cruciat CM, Remor M, Hofert C, Schelder M, Brajenovic M, Ruffner H, Merino A, Klein K, Hudak M, Dickson D, Rudi T, Gnau V, Bauch A, Bastuck S, Huhse B, Leutwein C, Heurtier MA, Copley RR, Edelmann A, Querfurth E, Rybin V, et al:: Functional organization of the yeast proteome by systematic analysis of protein complexes. Nature 2002, 415(6868):141-147.

6. Fu Y, Gu Y, Zheng Z, Wasteneys G, Yang Z: Arabidopsis interdigitating cell growth requires two antagonistic pathways with opposing action on cell morphogenesis. Cell 2005, 120(5):687-700.

7. Poethig RS: Phase change and the regulation of developmental timing in plants. Science 2003, 301(5631):334-336.

8. Li X, Zhang Y, Clarke JD, Li Y, Dong X: Identification and cloning of a negative regulator of systemic acquired resistance, $S N I 1$, through a screen for suppressors of npr1-1. Cell 1999, 98:329-339.

9. Berardini TZ, Bollman K, Sun H, Poethig RS: Regulation of vegetative phase change in Arabidopsis thaliana by cyclophilin 40. Science 2001, 291(5512):2405-2407.

10. Bollman KM, Aukerman MJ, Park MY, Hunter C, Berardini TZ, Poethig RS: HASTY, the Arabidopsis ortholog of exportin 5/MSN5, regulates phase change and morphogenesis. Development 2003, 130(8):1493-1504.

11. Hunter C, Sun H, Poethig RS: The Arabidopsis heterochronic gene ZIPPY is an ARGONAUTE family member. Curr Bio/ 2003, 13(19):1734-1739.

12. Peragine A, Yoshikawa M, WU G, Albrecht HL, Poethig RS: SGS3 and SGS2/ SDE1/RDR6 are required for juvenile development and the production of trans-acting siRNAs in Arabidopsis. Genes Dev 2004, 18(19):2368-2379.

13. Telfer A, Bollman KM, Poethig RS: Phase change and the regulation of trichome distribution in Arabidopsis thaliana. Development 1997 124:645-654.

14. Tsuge $T$, Tsukaya H, Uchimiya H: Two independent and polarized processes of cell elongation regulate leaf blade expansion in Arabidopsis thaliana (L) Heynh. Development 1996, 122(5):1589-1600.

15. Tsukaya H: Organ shape and size: a lesson from studies of leaf morphogenesis. Curr Opin Plant Biol 2003, 6(1):57-62.

16. Folkers U, Kirik V, Schobinger U, Falk S, Krishnakumar S, Pollock MA, Oppenheimer DG, Day I, Reddy AS, Jurgens G, Hulskamp M: The cell morphogenesis gene ANGUSTIFOLIA encodes a CtBP/BARS-like protein and is involved in the control of the microtubule cytoskeleton. Embo J 2002, 21(6):1280-1288.

17. Li H, Shen JJ, Zheng ZL, Lin Y, Yang Z: The Rop GTPase switch controls multiple developmental processes in Arabidopsis. Plant Physiol 2001, 126(2):670-684.

18. Alonso-Blanco C, Peeters A, Koornneef M, Lister C, Dean C, van den Bosch N, Pot J, Kuiper M: Development of an AFLP based linkage map of Ler, Col and Cvi Arabidopsis thaliana ecotypes and construction of a Ler/ Cvi recombinant inbred line population. Plant J 1998, 14:259-271.

19. Lee I, Aukerman MJ, Gore SL, Lohman KN, Michaels SD, Weaver LM, John MC, Feldmann KA, Amasino RM: Isolation of LUMINIDEPENDENS: a gene involved in the control of flowering time in Arabidopsis. Plant Cell 1994, 6(1):75-83.

20. Brune B, Gotz C, Mebmer UK, Sandau K, Hirvonen M-R, Lapentina EG: Superoxide formation and macrophage resistance to nitric oxidemediated apoptosis. J Biol Chem 1997, 272(11):7253-7258.

21. Lim MH, Kim J, Kim YS, Chung KS, Seo YH, Lee I, Kim J, Hong CB, Kim HJ, Park CM: A new Arabidopsis gene, FLK, encodes an RNA binding protein with $\mathrm{K}$ homology motifs and regulates flowering time via FLOWERING LOCUS C. Plant Cell 2004, 16(3):731-740.

22. Holub EB: The arms race is ancient history in Arabidopsis, the wildflower. Nat Rev Genet 2001, 2:1-12.

23. True JR, Carroll SB: Gene co-option in physiological and morphological evolution. Annu Rev Cell Dev Biol 2002, 18:53-80.

24. Slusarenko AJ, Schlaich NL: Downy mildew of Arabidopsis thaliana caused by Hyaloperonospora parasitica (formerly Peronospora parasitica). Molecular Plant Pathology 2003, 4(3):159-170.

25. Haas BJ, Kamoun S, Zody MC, Jiang RH, Handsaker RE, Cano LM, Grabherr M, Kodira CD, Raffaele S, Torto-Alalibo T, Bozkurt TO, Ah-Fong AM Alvarado L, Anderson VL, Armstrong MR, Avrova A, Baxter L, Beynon J, Boevink PC, Bollmann SR, Bos Jl, Bulone V, Cai G, Cakir C, Carrington JC, 
Chawner M, Conti L, Costanzo S, Ewan R, Fahlgren N, et al: Genome sequence and analysis of the Irish potato famine pathogen Phytophthora infestans. Nature 2009, 461(7262):393-398.

26. Dangl JL, Jones JDG: Plant pathogens and integrated defence responses to infection. Nature 2001, 411:826-833.

27. Jones JD, Dangl JL: The plant immune system. Nature 2006 444(7117):323-329.

28. Mourrain P, Beclin C, Elmayan T, Feuerbach F, Godon C, Morel JB, Jouette D, Lacombe AM, Nikic S, Picault N, Remoue K, Sanial M, Vo TA, Vaucheret H: Arabidopsis SGS2 and SGS3 genes are required for posttranscriptional gene silencing and natural virus resistance. Cell 2000, 101(5):533-542.

29. Prigge MJ, Wagner DR: The arabidopsis serrate gene encodes a zincfinger protein required for normal shoot development. Plant Cell 2001 13(6):1263-1279.

30. Earley KW, R. HJ, Pontes O, Opper K, Juehne T, Song K, S. P: Gatewaycompatible vectors for plant functional genomics and proteomics: pEarleyGates, pHellsgates and alternative destinations. submitted 2005.

31. Smyth G: Linear Models and Empirical Bayes Methods for Assessing Differential Expression in Microarray Experiments. Statistical Applications in Genetics and Molecular Biology 2004, 3:3-7.

32. Benjamini Y, Drai D, Elmer G, Kafkafi N, Golani I: Controlling the false discovery rate in behavior genetics research. Behav Brain Res 2001, 125(12):279-284.

doi: $10.1186 / 1471-2229-10-203$

Cite this article as: Tsuchiya and Eulgem, Co-option of EDM2 to distinct regulatory modules in Arabidopsis thaliana development BMC Plant Biology 2010, 10:203

\section{Submit your next manuscript to BioMed Central} and take full advantage of:

- Convenient online submission

- Thorough peer review

- No space constraints or color figure charges

- Immediate publication on acceptance

- Inclusion in PubMed, CAS, Scopus and Google Scholar

- Research which is freely available for redistribution

Submit your manuscript at www.biomedcentral.com/submit
C Biomed Central 